\title{
We Asked the Experts: "To Stent or Not to Stent... What is the Best Management of an Esophageal Leak or Benign Perforation?"
}

\author{
Sarah K. Thompson ${ }^{1}$ David I. Watson ${ }^{1}$
}

Published online: 3 February 2020

(C) Société Internationale de Chirurgie 2020

In November 2019, the Journal published an updated systemic review of the impact of esophageal stents in the management of esophageal anastomotic leaks and benign perforations [1]. Although only recently published online, this paper has generated much positive and negative feedback, perhaps magnified by the authors' use of 'Twitter'!

Since the first systematic review was published in 2014 (by many of the same authors [2]), the number of publications pertinent to this topic has more than doubled and the cumulative number of patients has increased from 340 to 1752 patients. Nevertheless, the results remain the same; metal stents perform better than plastic stents with a reduced risk of migration, perforation, and repositioning. The absolute percentage of technical and clinical success rates has also increased either reflecting improvements in stent design or greater experience in the insertion/removal of stents. But, has the review provided an answer to the surgeon's question when faced with the dreaded anastomotic leak, or a Boerhaave's perforation in the early hours of the morning? And, five years later, is stenting really the "go to" strategy for managing these complicated (and usually frail and/or de-conditioned) patients?

An opposing viewpoint from a high-volume UK esophageal cancer unit was published in the British Journal of Surgery in 2016 [3]. The authors of that paper retrospectively reviewed 31 patients who developed a leak following esophagectomy ( $8 \%$ of 390 esophagectomies over a 5 -year period). Twenty-seven patients were managed non-

Sarah K. Thompson

sarah.thompson@flinders.edu.au

1 Department of Surgery, Rm 3D204, Flinders Medical Centre, Flinders University Discipline of Surgery, Bedford Park, SA 5042, Australia operatively with nasogastric tube decompression, antimicrobials including antifungals, enteral nutrition, and in one third, radiological drains were inserted. One patient required surgery for worsening sepsis, and two had late stent insertion to facilitate oral feeding. Significantly, there were no deaths in this series! It is difficult to compare their mortality rate to the $7 \%$ reported in the most recent systematic review above, as benign esophageal perforations were combined, but stent erosion in of itself does appear to carry a significant mortality risk [1].

Enter the "new kid on the block." Endoscopic vacuum therapy (EVT) involves the insertion of a nasogastric tube with a porous polyurethane sponge attached to its distal end through the leak and into the adjacent cavity, and then connected to a continuous negative pressure pump, thereby aiming to collapse the cavity [4]. Weidenhagen et al. first published this technique to treat a leak following rectal surgery. It was then applied to leaks of the upper gastrointestinal tract by Loske and Müller in 2006, and the first commercial system was eventually developed in 2014 (EsoSPONGE®, B. Braun Melsungen AG, Melsungen, Germany) [4]. EVT remains the common acronym for the technique even though EndoVac, E-Vac, and endoscopic negative pressure therapy (ENPT) are also in use. EVT appears effective as it improves perfusion, reduces edema, removes secretions, and encourages the formation of granulation tissue. An advantage over other therapies is the simultaneous closure of the defect and internal drainage of the leak, obviating the need for external drainage [4].

The first systematic review and meta-analysis comparing self-expanding metal stents to EVT was published in 2018 by Bonavina et al. [5]. Four observational studies (three from Germany and one from Korea) were included with a total patient number of 163 . They found that EVT had a higher success rate in healing esophageal leaks, a 
lower major complication rate, and a lower in-hospital mortality rate when compared to stents.

A "We Asked the Experts" column would not be complete without a comment on our practice! When we undertake esophagectomy, we work on the assumption that an anastomotic leak will occur, i.e., prepare for the worst and hope for the best! We place drains to cover the anastomosis, with at least one drain remaining in place until we are confident that the anastomosis has healed. We also routinely place a jejunostomy tube for early feeding, and as insurance in case of a leak. If we suspect a leak, a CT scan with oral contrast is requested to confirm the suspicion and check whether the surgical drain(s) is providing adequate drainage. For most leaks (type I or II), our preference is non-operative management as described in Griffin et al's paper, with antimicrobials, antifungals, and enteral feeding. However, if severe sepsis and/or extensive contamination are present, operative management is needed (type III leak). In general, if a type I or II leak is well-drained by the original surgical drain(s), conservative management will be successful. If an undrained collection is present, then we might consider EVT rather than stent for the reasons described above.

Over the last decade, the authors have personally performed 204 esophagectomies without mortality, and anastomotic leaks occurred following 14 (6.9\%) of these procedures. Nine $(4.4 \%)$ of the leaks were managed conservatively, and five $(2.5 \%)$ required operative re-intervention. We did not use stent or EVT for the management of any of these patients. However, we have used EVT for the management of selected patients with benign esophageal perforations. The decision to use EVT rather than surgery hinged upon whether there was a breach of the pleura with contamination of a pleural cavity. If yes, a thoracotomy is our preferred approach with repair of the esophageal tear, washout of the affected pleural cavity, placement of drains, and establishment of feeding access. If not, we have used EVT. For iatrogenic perforations where mediastinal contamination has been relatively contained (e.g., after endoscopic dilatation, or from placement of an esophageal probe for transesophageal echocardiography), EVT has generally been successful; although when contamination is more extensive (e.g., some Boerhaave's syndrome), EVT might not deliver adequate drainage. When used successfully, patients have generally required between 6-8 sponge changes at an interval of every 3 to 4 days. Drainage is continued until the cavity has sealed off and collapsed.

So, what is the best management strategy for an esophageal leak or perforation? As alluded to by Bonavina et al. [5], there is no common algorithm for the management of esophageal anastomotic leak or benign perforation. To facilitate international consensus for best management of leak, future studies should adhere to a standardized definition of post-esophagectomy leak, ideally the system established by the Esophagectomy Complications Consensus Group [3, 5]. We support Griffiths et al. view that randomized trials comparing stents to EVT are desperately needed to provide good quality data. As meta-analyses that incorporate poor quality data are potentially misleading, a meeting between internationally recognized experts in the field of esophageal disease should be considered to develop guidelines for the management of esophageal leak/perforation. We thank the editors for asking us to comment on this important topic.

\section{References}

1. Kamarajah SK, Bundred J, Spence G et al (2019) Critical appraisal of the impact of oesophageal stents in the management of oesophageal anastomotic leaks and benign oesophageal perforations: an updated systematic review. World J Surg. https://doi.org/ 10.1007/s00268-019-05259-6

2. Dasari BV, Neely D, Kennedy A et al (2014) The role of esophageal stents in the management of esophageal anastomotic leaks and benign esophageal perforations. Ann Surg 259:852-860

3. Dent B, Griffin SM, Jones R et al (2016) Management and outcomes of anastomotic leaks after oesophagectomy. Br J Surg 103:1033-1038

4. Loske G (2019) Endoscopic negative pressure therapy of the upper gastrointestinal tract. Chirurg 90:1-6

5. Rausa E, Asti E, Aiolfi A et al (2018) Comparison of endoscopic vacuum therapy versus endoscopic stenting for esophageal leaks: a systematic review and meta-analysis. Dis Esophagus. https://doi. org/10.1093/dote/doy060

Publisher's Note Springer Nature remains neutral with regard to jurisdictional claims in published maps and institutional affiliations. 